\title{
RELACIÓN ENTRE EL AUTOCONCEPTO Y EL RENDIMIENTO ACADÉMICO EN ADOLESCENTES
}

Marina Agapito Romero, Ana Calderón Callejo, Blanca Cobo Jarones y Laura Rodríguez Rodríguez. Universidad de Huelva

\section{RESUMEN}

El estudio presente se centra en las relaciones existentes entre el autoconcepto y el rendimiento académico. Se ha elegido una muestra aleatoria de 67 adolescentes entre 14 y 15 años. Mediante la prueba estandarizada "Autoconcepto resultante del entorno social: Cuestionarios SC1 de Brookover y SC2; SC3 y SC4 de Coopersmith (1982)", comparada con las notas obtenidas en el anterior trimestre, se ha podido observas cómo se correlaciona la opinión que tiene el adolescente de sí mismo y las notas que finalmente obtienen. Asimismo, apreciamos la influencia que ejerce sobre el autoconcepto de dichos adolescentes la opinión de padres y profesores.

Palabras clave: Autoconcepto, rendimiento académico, calificaciones, adolescentes

\section{INTRODUCCIÓN}

El autoconcepto juega un papel fundamental en el desarrollo de la personalidad. Un autoconcepto positivo está en la base del buen funcionamiento personal, social y profesional dependiendo de él, la satisfacción personal, el sentirse bien consigo mismo (Goñi, E.,2009), y como se pretende comprobar aquí, un buen rendimiento académico. De ahí que el logro de un equilibrio socioafectivo en el alumnado a partir de una imagen ajustada y positiva de sí mismo figure entre las finalidades tanto de la Educación Primaria como de la Educación Secundaria Obligatoria (Goñi, E.,2009).

A mediados de los años setenta, se produce un cambio en la forma de entender el autoconcepto, al ser reemplazada una visión unidimensional por una concepción jerárquica y multidimensional. El autoconcepto global vendría a ser el resultado de un conjunto de percepciones parciales del propio yo, que se estructuran en una organización jerárquica: el autoconcepto general se compondría del autoconcepto académico y del no-académico y este último, a su vez, incluiría tanto el autoconcepto social como el personal y el físico (Goñi, E.,2009). Aquí se intentará hacer una pequeña prueba de la correlación existente entre algunos aspectos del autoconcepto general.

En un estudio realizado en la universidad de Granada por María Tamara Polo Sánchez y María Dolores López-Justicia en el que vincularon autoconcepto académico y discapacidad en la universidad, obtuvieron resultados que confirmaron que la presencia de la discapacidad parece asociada con un nivel más bajo de autoconcepto académico y emocional, esto es, los estudiantes con discapacidad se perciben menos competentes en el ámbito académico (Polo, M.T. y López-Justicia, M.D., 2009). Aunque la discapacidad no es el tema de este estudio, parece interesante cómo la autopercepción influye de manera directa con el rendimiento.

Otro estudio realizado en la universidad Miguel Hernández de Elche, ha examinado las variables que se relacionan con el rendimiento académico en los adolescentes con

1 ReiDoCrea. Revista electrónica de investigación Docencia Creativa. Volumen 2. Páginas 181-187 
padres divorciados. Los adolescentes con padres divorciados obtienen más suspensos que los que tienen padres casados (Orgilés, M., Johnson, B.T., Huedo-Medina, T.B., Espada, J.P., 2012). Este hallazgo se relaciona con resultados de estudios previos que indican que los adolescentes con padres divorciados tienen peor rendimiento académico que aquellos cuyos padres viven juntos (e.g. Tillman, 2007). Los adolescentes con padres divorciados tienen también peor autoconcepto que el de aquellos que proceden de familias con padres casados (e.g., Sweeney y Bracken, 2000).

\section{MÉTODO}

\section{Hipótesis}

- Lo que los alumnos piensan sobre sí mismos influye sobre las notas. A mayor puntuación en las escala SC1 y SC2, mejores resultados académicos.

- Lo que padres y profesores opinan sobre los alumnos correlaciona directamente con lo que opinan los alumnos sobre sí mismos.

- De igual manera, las puntuaciones obtenidas en las escalas SC3 y SC4, correlacionan directamente con los resultados académicos. Es decir, lo que opinen padres y profesores también influye de forma directa con los resultados académicos de los alumnos.

- Las notas obtenidas están relacionadas directamente con el autoconcepto. Altas puntaciones en autoconcepto se correlacionan con buenos resultados académicos.

\section{Sujetos}

Los participantes de este estudio han sido 67 alumnos del Colegio Diocesano Sagrado Corazón de Jesús de Huelva, de tres clases diferentes del mismo curso, que se encuentran cursando $3^{\circ}$ de Educación Secundaria en el año 2013, y tienen edades comprendidas entre 14 y 15 años.

\section{Instrumentos}

Se ha utilizado el cuestionario Autoconcepto resultante del entorno social: Cuestionarios SC1 de Brookover y SC2; SC3 y SC4 de Coopersmith (1982), en el que han sido consideradas cuatro subvariables, para medir el autoconcepto, con cuatro puntuaciones independientes que corresponden a las siguientes variables $e$ instrumentos:

SC1 de autoconcepto general, bajo el título “¿Cómo soy?”, es la adaptación (Self Concept) de Rodríguez Espinar, S. del denominado S.E.I. (Self-Steem Inventory) de Stanley Coopersmith, en su forma reducida de 25 ítems. (Purkey, 1.968 en Rodríguez Espinar, S., 1.982, p.78) quien la confeccionó a partir de otras escalas más amplias de Rogers y Dymond con la que guarda una correlación de 0,86 . El sujeto responde con verdadero o falso de acuerdo con lo que el sujeto opina de sí mismo.

Fiabilidad: Coopersmith obtuvo valores de 0,88 para un intervalo de cinco semanas y de una muestra de alumnos de $5^{\circ}$ grado. Rodríguez Espinar obtuvo un valor de 0,86 con alumnos de $8^{\circ}$ de E.G.B. en el año 1.982 para un intervalo de un mes. 
SC2 de autoconcepto académico con el título “¿Qué opinas de ti mismo?”, adaptación de Rodríguez Espinar, S. para su investigación "Factores de Rendimiento Escolar"(1.982) de la escala Self-Concept of Hability General desarrollado por Brookover et al (1.967) para alumnos de los cursos $7^{\circ}-12^{\circ}$ del sistema educativo norteamericano y que consta de una serie de 7 ítems para cada individuo que opina de sí mismo en el presente y futuro en lo puramente académico. Mediante un valor categorizado de 1 a 5 en cada uno de ellos resultado de elegir una opción y que puede dar una puntuación máxima de 35 y mínima de 7.

Fiabilidad: Brookover et al (1.974), obtuvieron valores de 0,82 en género masculino y 0,77 en el femenino en $7^{\circ}$ curso y para grupos iguales de $8^{\circ}$ de 0,85 y 0,87 respectivamente. La adaptación de Rodríguez Espinar (1.982) obtuvo en una muestra combinada de alumnos de Segunda Etapa de un valor de 0,82.

EI SC3 de autoconcepto académico titulado “¿Qué opinan tus padres de ti?" y el SC4 de autoconcepto académico titulado “¿Qué opina tu profesor de ti?" corresponden a las variables del autoconcepto académico percibido en las personas de los padres y el profesor respectivamente. Asumidas por las escalas Perceived Parental Evaluations of Ability y Perceived Teachers' Evaluations of Ability, desarrolladas también por Brookover y colaboradores. Cada una de ellas posee cinco ítems y, como en el SC2, con el mismo tipo de aplicación y puntuación.

Fiabilidad: Brookover y sus colaboradores para una muestra de 500 alumnos norteamericanos de $8^{\circ}$ curso, obtuvieron unos valores de 0,84 y 0,92 respectivamente. Y Rodríguez Espinar para 100 alumnos de Segunda Etapa españoles, obtuvo valores análogos de 0,83 y 0.90. (Périz, J.A., 2007)

Además, se ha añadido un apartado, donde se preguntaba a los alumnos los resultados académicos que suelen obtener en cada una de las materias. Después, se ha hecho una media de las notas obtenidas entre todas las materias. Para posteriormente, poder hacer la correlación entre autoconcepto y resultado académico.

\section{Procedimiento}

Para realizar el cuestionario, nos pusimos en contacto con la directora del Colegio Diocesano Sagrado Corazón de Jesús de Huelva. Tras el consentimiento, se realizó el cuestionario a 67 alumnos, de los cuales 27 eran chicos (40.3\%) y 40 chicas $(59.7$ $\%)$.

\begin{tabular}{llllll} 
Sexo & & & & \\
\hline & & Frecuencia & Porcentaje & Porcentaje válido & $\begin{array}{l}\text { Porcentaje } \\
\text { acumulado }\end{array}$ \\
\hline \multirow{2}{*}{ Válidos } & Chico & 27 & 40,3 & 40,3 & 40,3 \\
& Chica & 40 & 59,7 & 59,7 & 100,0 \\
& Total & 67 & 100,0 & 100,0 & \\
\hline
\end{tabular}

\section{Análisis estadístico}

Con ayuda del programa estadístico SPSS 15.0, calculamos correlaciones no paramétricas. En primer lugar, se ha realizado un coeficiente de correlación de Spearman. Posteriormente, se han realizado otros cálculos para comprobar otros datos de interés que hayan podido pasar desapercibidos en una primera aproximación. Las pruebas realizadas han sido, correlaciones bivariadas y ANOVA de un factor. 


\section{RESULTADOS}

\section{Lo que los alumnos piensan sobre sí mismos influye sobre las notas.}

Para contrastar esta hipótesis se ha realizado el siguiente estadístico con el programa SPSS.

\begin{tabular}{|c|c|c|c|c|c|}
\hline \multicolumn{6}{|c|}{ Correlaciones } \\
\hline & & & SC1 & $\mathrm{SC} 2$ & Nota \\
\hline \multirow[t]{9}{*}{ Rho de Spearman } & $\mathrm{SC} 1$ & $\begin{array}{l}\text { Coeficiente de } \\
\text { correlación }\end{array}$ & 1,000 &, 153 &, 058 \\
\hline & & Sig. (bilateral) & . &, 215 & 639 \\
\hline & & $N$ & 67 & 67 & 67 \\
\hline & $\mathrm{SC} 2$ & $\begin{array}{l}\text { Coeficiente de } \\
\text { correlación }\end{array}$ &, 153 & 1,000 &, $664^{n}$ \\
\hline & & Sig. (bilateral) &, 215 & . &, 000 \\
\hline & & $N$ & 67 & 67 & 67 \\
\hline & Nota & $\begin{array}{l}\text { Coeficiente de } \\
\text { correlación }\end{array}$ & ,058 &, $664^{\pi m}$ & 1,000 \\
\hline & & Sig. (bilateral) & 639 &, 000 & . \\
\hline & & $\mathrm{N}$ & 67 & 67 & 67 \\
\hline
\end{tabular}

Si observamos la tabla de la correlación de Spearman, observamos que no hay correlación significativa entre el SC1 (¿Cómo soy?) y las notas obtenidas. Sin embargo, si encontramos una relación bastante significativa entre lo que los alumnos opinan de sí mismos (SC2) y los resultados académicos. Es decir, mayores puntuaciones en el cuestionario SC1 no influyen sobre las notas obtenidas, pero a mayor puntuación obtenida en el cuestionario SC2, mejores resultados académicos obtendrá el alumno. Por tanto, se podría decir que nuestra hipótesis se cumple solo en parte, ya que, según estos resultados, sobre el resultado académico influye la percepción que tiene uno de sí mismo, pero no influye el cómo se es.

\section{Lo que padres y profesores opinan sobre los alumnos correlaciona directamente con lo que opinan los alumnos sobre sí mismos.}

Al igual que en el caso anterior, vemos que no existe una relación significativa entre los resultados obtenidos en el cuestionario SC1 y los otros cuestionarios. Sin embargo, si observamos las relaciones del SC2 (¿qué opinas de ti mismo?) con el SC3 (¿Qué opinan tus padres de ti?) y SC4 (¿qué opina tu profesor/a de ti?), observamos que existe una relación muy significativa. Es decir, se cumple nuestra hipótesis, la opinión de padres y profesores sobre el alumno, influye en la opinión de este sobre sí mismo. Otro dato curioso, obtenido al realizar estos estadísticos, es la relación entre SC3 y SC4, podríamos deducir, que existe una relación directamente proporcional entre la opinión de padres y profesores, es decir, a mejor opinión tengan los profesores sobre los alumnos, mejor opinión tienen los padres, y viceversa. 
Lo que opinen padres y profesores también influye de forma directa con los resultados académicos de los alumnos.

\begin{tabular}{|c|c|c|c|c|c|}
\hline \multicolumn{6}{|c|}{ Correlaciones } \\
\hline & & & $\mathrm{SC} 3$ & $\mathrm{SC} 4$ & Nota \\
\hline \multirow[t]{9}{*}{ Rho de Spearman } & $\mathrm{SC} 3$ & $\begin{array}{l}\text { Coeficiente de } \\
\text { correlación }\end{array}$ & 1,000 & $689^{\prime \prime \prime}$ & $.623^{\prime \prime}$ \\
\hline & & Sig. (bilateral) & . &, 000 &, 000 \\
\hline & & N & 67 & 67 & 67 \\
\hline & $\mathrm{SC} 4$ & $\begin{array}{l}\text { Coeficiente de } \\
\text { correlación }\end{array}$ & $689^{m}$ & 1,000 &, $488^{11}$ \\
\hline & & Sig. (bilateral) & , 000 & &, 000 \\
\hline & & N & 67 & 67 & 67 \\
\hline & Nota & $\begin{array}{l}\text { Coeficiente de } \\
\text { correlación }\end{array}$ & $.623^{\mathrm{Nm}}$ &, $488^{\mathrm{nN}}$ & 1,000 \\
\hline & & Sig. (bilateral) &, 000 &, 000 & . \\
\hline & & N & 67 & 67 & 67 \\
\hline
\end{tabular}

De la misma manera, comprobamos que existe correlación muy significativa entre SC3 (opinión de los padres) y SC4 (opinión de los profesores) y las notas obtenidas. Es decir, a mejor opinión de padres y profesores, mejores notas obtienen los alumnos. Por tanto, también se verifica nuestra hipótesis.

\section{Las notas obtenidas están relacionadas directamente con el autoconcepto.}

Con los resultados obtenidos anteriormente, podemos concluir que se verifica nuestra hipótesis general. El autoconcepto se relaciona de forma directamente proporcional con el resultado académico. Es decir, a mejor autoconcepto, incluyendo lo que uno piensa sobre sí mismo, y lo que piensan padres y profesores, mejores resultados académicos obtendrán los alumnos.

\section{DISCUSIÓN}

El presente trabajo pretendía valorar la relación existente entre el autoconcepto y las notas obtenidas. El cuestionario se adecuó correctamente a los sujetos estudiados y los grupos y, al ser de la misma edad, pudieron ser comparados entre sí.

Se ha podido comprobar, tras realizar un análisis y verificar las hipótesis, que mayores puntuaciones en el cuestionario sobre cómo soy, no influyen sobre las notas obtenidas, pero a mayor puntuación en el cuestionario sobre la opinión de sí mismo, mejores resultados académicos. Esto podría abrir un nuevo tema de debate para futuras investigaciones. A su vez, la visión de sus padres y de sus profesores influye de forma significativa en la percepción que tienen. Esto es, la opinión que tienen los padres y profesores influye en la opinión sobre ellos mismos.

Es posible que las puntuaciones obtenidas en el SC1 no influyan en las calificaciones, debido a la distinción que se realiza en el cuestionario. ¿Cómo soy? y ¿Qué opinas de 
ti mismo? son dos ítems que tienen relación entre sí, pero no valoran lo mismo. El primer ítem está relacionado con la forma de ser de cada uno, de forma general, y el segundo se refiere a la opinión que se tiene de sí mismos, es decir, puedes ser inteligente (ítem 1) pero percibirlo de forma negativa (ítem 2, opinión de sí mismos).

Para experimentos posteriores, una propuesta podría ser no realizar esta última distinción, ya que puede llevar a confusión por la fina línea divisoria entre ambos conceptos.

Al realizar una ANOVA de un factor, se pudo observar otro dato que parece interesante, aunque no se presenta entre nuestras hipótesis. No se encuentra ninguna diferencia significativa en cuanto al sexo, en ninguna de las variables.

ANOVA de un factor

\begin{tabular}{|c|c|c|c|c|c|c|}
\hline & & $\begin{array}{l}\text { Suma de } \\
\text { cuadrados }\end{array}$ & $\mathrm{gl}$ & $\begin{array}{c}\text { Media } \\
\text { cuadrática }\end{array}$ & $\mathrm{F}$ & Sig. \\
\hline \multirow[t]{3}{*}{ SC1 } & Inter-grupos & 2,080 & 1 & 2,080 & 166 &, 685 \\
\hline & Intra-grupos & 814,785 & 65 & 12,535 & & \\
\hline & Total & 816,866 & 66 & & & \\
\hline \multirow[t]{3}{*}{$\mathrm{SC} 2$} & Inter-grupos & 1,882 & 1 & 1,882 & ,073 & ,789 \\
\hline & Intra-grupos & 1686,775 & 65 & 25,950 & & \\
\hline & Total & 1688,657 & 66 & & & \\
\hline \multirow[t]{3}{*}{$\mathrm{SC} 3$} & Inter-grupos & 13,682 & 1 & 13,682 & 1,003 & 320 \\
\hline & Intra-grupos & 887,005 & 65 & 13,646 & & \\
\hline & Total & 900,687 & 66 & & & \\
\hline \multirow[t]{3}{*}{$\mathrm{SC} 4$} & Inter-grupos & 192 & 1 & 192 & ,016 & ,901 \\
\hline & Intra-grupos & 794,285 & 65 & 12,220 & & \\
\hline & Total & 794,478 & 66 & & & \\
\hline
\end{tabular}

\begin{tabular}{|c|c|c|c|c|c|c|c|c|c|}
\hline \multicolumn{10}{|c|}{ Descriptivos } \\
\hline & & \multirow[b]{2}{*}{$\mathrm{N}$} & \multirow[b]{2}{*}{ Media } & \multirow[b]{2}{*}{$\begin{array}{l}\text { Desviación } \\
\text { típica }\end{array}$} & \multirow[b]{2}{*}{ Error típico } & \multicolumn{2}{|c|}{$\begin{array}{l}\text { Intervalo de confianza para la } \\
\text { media al } 95 \%\end{array}$} & \multirow[b]{2}{*}{ Mínimo } & \multirow[b]{2}{*}{ Máximo } \\
\hline & & & & & & Limite inferior & $\begin{array}{l}\text { Límite } \\
\text { superior }\end{array}$ & & \\
\hline \multirow[t]{3}{*}{ SC1 } & Chico & 27 & 14,26 & 3,096 &, 596 & 13,03 & 15,48 & 8 & 19 \\
\hline & Chica & 40 & 13,90 & 3,808 &, 602 & 12,68 & 15,12 & 6 & 21 \\
\hline & Total & 67 & 14,04 & 3,518 & ,430 & 13,19 & 14,90 & 6 & 21 \\
\hline \multirow[t]{3}{*}{$\mathrm{SC} 2$} & Chico & 27 & 23,33 & 5,870 & 1,130 & 21,01 & 25,66 & 14 & 35 \\
\hline & Chica & 40 & 23,68 & 4,503 &, 712 & 22,23 & 25,12 & 12 & 32 \\
\hline & Total & 67 & 23,54 & 5,058 & ,618 & 22,30 & 24,77 & 12 & 35 \\
\hline \multirow[t]{3}{*}{$\mathrm{SC} 3$} & Chico & 27 & 18,70 & 3,383 & .651 & 17,37 & 20,04 & 13 & 25 \\
\hline & Chica & 40 & 19,63 & 3,887 &, 615 & 18,38 & 20,87 & 6 & 25 \\
\hline & Total & 67 & 19,25 & 3,694 & ,451 & 18,35 & 20,15 & 6 & 25 \\
\hline \multirow[t]{3}{*}{$\mathrm{SC} 4$} & Chico & 27 & 18,26 & 3,938 & ,758 & 16,70 & 19,82 & 10 & 26 \\
\hline & Chica & 40 & 18,15 & 3,167 &, 501 & 17,14 & 19,16 & 12 & 25 \\
\hline & Total & 67 & 18,19 & 3,470 &, 424 & 17,35 & 19,04 & 10 & 26 \\
\hline
\end{tabular}

Esta investigación pone de manifiesto que en las instituciones es importante trabajar el autoconcepto, ya que como se ha comprobado, influye de forma significativa. En numerosas ocasiones, ante el fracaso escolar, se pretende disminuir este número de suspensos con clases particulares o refuerzos sin hacer mención o tener en cuenta la percepción que tienen los alumnos sobre sí mismos y cómo puede influir en sus calificaciones. Por ello, puede ser considerado necesario realizar intervenciones sobre este aspecto, e investigar si al realizar actividades que mejoren el autoconcepto, se mejora el rendimiento académico, sería un aspecto a valorar muy interesante que completaría este estudio. 


\section{REFERENCIAS}

Brookover, W.B. et al (1.974) "Self-concept of ability and school achievement". Sociology of Education $n^{\circ} 73$, pp. (271-278)

Goñi, E., Madariaga, J.M. (2009) "El autoconcepto personal: estructura interna, medida y variabilidad". Servicio Editorial de la Universidad del País Vasco Euskal Herriko Unibertsitateko Argitalpen Zerbitzua.

Orgilés, M., Johnson, B.T., Huedo-Medina, T.B., Espada,J.P., (2012). "Autoconcepto y ansiedad social como variables predictoras del rendimiento académico de los adolescentes españoles con padres divorciados." Electronic Journal of Research in Educational Psychology, 10 (1), 057-072.

Périz, J.A., Martínez, M.C., Generelo, E. (2007) "Influencia de las variables bio-psicosociales de salud en la adherencia a la práctica físico-deportiva en adolescentes (1316 años)". Departamento de Educación, Cultura y Deporte: Aragón.

Polo, M.T. y López-Justicia, M.D., (2009) "Autoconcepto de estudiantes universitarios con discapacidad visual, auditiva y motora." Revista Latinoamericana de Psicología 44 (2), 87-98

Purkey, W.W. (1.968). "The search for self: evaluating students self concept" en Research Bulletin. Florida: Educational Research and Development Council 4, $n^{\circ} 2$.

Sánchez García, M. y Carmona Márquez, J. (2010) "Análisis de datos con SPSS 12". Universidad de Huelva Publicaciones.

Santana Vega, L. E. y Feliciano, L. (2011). Percepción de apoyo de padres y profesores, autoconcepto y toma de decisiones en bachillerato Revista de Educación, 355, 493-519.

Sweeney, R.B., y Bracken, B.A. (2000). Influence of family structure on children's selfconcept development. Canadian Journal of School Psychology, 16(1), 39-52.

Tillman, K.H. (2007). Family structure pathways and academic disadvantage amogn adolescents in stepfamilies. Sociological Inquiry, 77, 383-424. 